\title{
Observations of Cold Antihydrogen
}

\author{
Gerald Gabrielse ${ }^{1}$ \\ Department of Physics, Harvard University, Cambridge, MA 02138
}

\begin{abstract}
ATRAP's $\mathrm{e}^{+}$cooling of $\overline{\mathrm{p}}$ in a nested Penning trap has led to reports of cold $\overline{\mathrm{H}}$ produced during such cooling by the ATHENA and ATRAP collaborations. ATHENA uses coincident annihilation detection and ATRAP uses field ionization followed by $\overline{\mathrm{p}}$ storage. Advantages of the later method include the complete absence of a substantial background of false events intrinsic to the former, and the first way to measure which $\overline{\mathrm{H}}$ states are produced. ATRAP enhances the $\overline{\mathrm{H}}$ production rate by driving many cycles of $\mathrm{e}^{+}$cooling in the nested trap, with more $\overline{\mathrm{H}}$ counted in an hour than the sum of all the other antimatter atoms ever reported. The number of $\overline{\mathrm{H}}$ counted per incident high energy $\overline{\mathrm{p}}$ is also higher than ever observed. The first measured distribution of $\overline{\mathrm{H}}$ states is made using a pre-ionizing electric field between separated production and detection regions. The high rate and the high Rydberg states suggest that the $\overline{\mathrm{H}}$ is formed via three-body recombination, as expected.
\end{abstract}

\section{Introduction}

Fourteen years ago, shortly after our TRAP team captured the first cold $\bar{p}$, I hesitated before putting into print the futuristic goal of using these to form $\overline{\mathrm{H}}$ that was cold enough to confine in a magnetic trap for precise laser spectroscopy [1]. In the intervening years, however, our TRAP Collaboration developed the techniques to slow, capture, cool and stack suitable cold $\overline{\mathrm{p}}$ at $4 \mathrm{~K}[2,3]$, an energy more than $10^{10}$ times lower than any other source. These techniques made possible a precise comparison of the charge-to-mass ratios of the $\bar{p}$ and $p$ that eventually reached an accuracy of 90 ppt [2], the most precise test of CPT invariance with baryons by many orders of magnitude. Meanwhile, some high velocity $\overline{\mathrm{H}}$ were observed $[4,5]$, but these cannot be easily used for precise studies.

Although no one predicted this, a new CERN storage ring (the Antiproton Decelerator, or $\mathrm{AD}$ ) now operates to allow two international collaborations to use these techniques to pursue cold $\overline{\mathrm{H}}$. ATRAP (which grew out of TRAP) demonstrated the crucial device and technique for producing cold antihydrogen about a year ago - the $\mathrm{e}^{+}$cooling of $\overline{\mathrm{p}}$ in a nested Penning trap [6]. This device and technique were then used to observe cold $\overline{\mathrm{H}}$, resulting in three recent reports of the observation cold $\overline{\mathrm{H}}-$ one by ATHENA [7] and two by ATRAP [8, 9].

The observations of cold $\overline{\mathrm{H}}$ start an exciting time, though much remains to be done. $\overline{\mathrm{H}}$ atoms that are cold enough to be trapped for laser spectroscopy promise to provide the most stringent CPT tests with baryons and leptons [2], along with more sensitive tests for possible extensions to the standard model [10], building on the high accuracy of hydrogen spectroscopy [11]. It may even be possible to directly observe the gravitational force on antimatter atoms $[12] . \overline{\mathrm{H}}$ atoms with a temperature near to the $0.5 \mathrm{~K}$ depth of a realistic magnetic trap are required if atoms are to be trapped from a thermal distribution with a reasonable efficiency.

${ }^{1}$ ATRAP Spokesperson, gabrielse@physics.harvard.edu 


\section{ATRAP's $\mathrm{e}^{+}$Cooling of $\overline{\mathrm{p}}$ in a Nested Penning Trap Produces Cold $\overline{\mathrm{H}}$}

The crucial step of demonstrating a way to produce cold $\overline{\mathrm{H}}$ was reported about a year ago when our ATRAP Collaboration demonstrated the first $\mathrm{e}^{+}$cooling of $\overline{\mathrm{p}}$ within a nested Penning trap [6]. The report suggested that the low relative velocity of $\bar{p}$ and $\mathrm{e}^{+}$made it likely that $\overline{\mathrm{H}}$ was produced. Indeed it was, as indicated by the recent reports of observed $\overline{\mathrm{H}}$ by ATHENA [7] and ATRAP [8, 9].

The key device allowing $\overline{\mathrm{H}}$ production is the nested Penning trap (Fig. 1) which we proposed long ago [13] as a way to allow $\mathrm{e}^{+}$and $\overline{\mathrm{p}}$ to interact while stored, despite the opposite sign of charge which keeps them from being confined together in the same Penning trap well. The $\mathrm{e}^{+}$are stored within a small inverted well at the center of a larger well for $\overline{\mathrm{p}}$. We investigated the nested Penning trap with $\mathrm{e}^{-}$and $\mathrm{p}$ [14], and loaded cold $\overline{\mathrm{p}}$ and $\mathrm{e}^{+}$together within such a trap [15], before using it to observe the $\mathrm{e}^{+}$cooling of $\overline{\mathrm{p}}$.

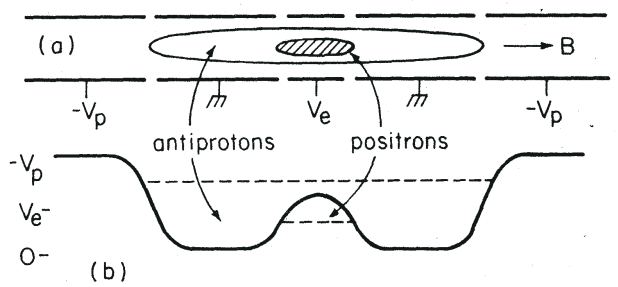

Figure 1: Nested Penning trap allows positrons and antiprotons to interact at low relative velocity. (From Ref. [13].)

The key process for $\overline{\mathrm{H}}$ production is the $\mathrm{e}^{+}$cooling of $\overline{\mathrm{p}}$ in the nested Penning trap, since this cooling produces $\overline{\mathrm{p}}$ and $\mathrm{e}^{+}$with a low relative velocity, the condition under which $\overline{\mathrm{H}}$ production is expected to be optimal. The $\overline{\mathrm{p}}$ start with high enough energies to take them back and forth across the whole width of their well. They loose energy via collisions while passing through the $\mathrm{e}^{+}$. The cooling gets more efficient as the $\overline{\mathrm{p}}$ energy declines to near where the $\overline{\mathrm{p}}$ and $\mathrm{e}^{+}$energies coincide, where the two species have low relative velocities. The long interaction time that results is what is desired for efficient $\overline{\mathrm{H}}$ production.

Fig. 2a shows an example of the energy of $\bar{p}$ in a nested Penning trap decreasing exponentially as a result of collisions with cold $\mathrm{e}^{+}$. The $\overline{\mathrm{p}}$ energy spectra at a sequence of cooling times (Fig. 2b-e) reveal intricate structure, not yet completely understood. $\overline{\mathrm{H}}$ presumably forms near to where the energies of the $\overline{\mathrm{p}}$ (histograms) and $\mathrm{e}^{+}$(vertical dashed line) overlap, where the relative velocities are low.

On a ten times longer time scale, ATRAP has also observed that the $\bar{p}$ cool into the side wells of the nested trap, decreasing the interaction with the $\mathrm{e}^{+}$. The new cooling mechanism here seems to be a recycled evaporative cooling of the $\bar{p}$, whereby hot $\overline{\mathrm{p}}$ that "evaporate" to higher energies in the nested well are cooled by the $\mathrm{e}^{+}$before they can leave the well. With no $\mathrm{e}^{+}$in the nested well, evaporative cooling cools the $\overline{\mathrm{p}}$ on the slower time scale.

ATRAP carefully studied $\overline{\mathrm{p}}$ losses from the nested Penning trap, and various in- 

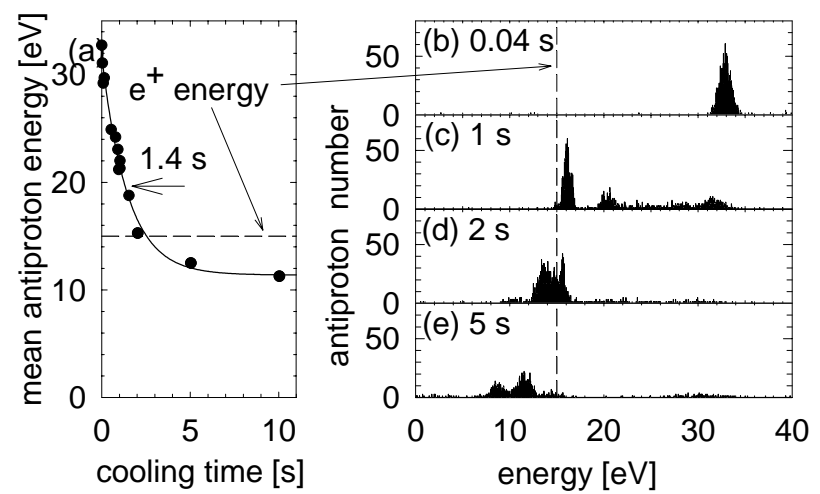

Figure 2: (a) Antiproton average energy decreases exponentially in time until the $\overline{\mathrm{p}}$ and $\mathrm{e}^{+}$have the lowest relative velocity. Cooling then continues but at a 10 times slower rate. (b-e) Energy spectra of the $\bar{p}$ as a function of the positron cooling time. (Here $5000 \overline{\mathrm{p}}$ are used, along with $200000 \mathrm{e}^{+}$in a $15 \mathrm{~V}$ well. From Ref. [8].)

triguing signals that seemed related to $\overline{\mathrm{H}}$ production. The concern was $\overline{\mathrm{p}}$ loss when $\overline{\mathrm{p}}$ energy is decreased to where they move slowly as they pass over the inverted $\mathrm{e}^{+}$well. Our detectors revealed radial losses taking place with $\mathrm{e}^{+}$in the nested well, but also without. The $\mathrm{e}^{+}$cooling brings the $\overline{\mathrm{p}}$ down to the energies where losses occur more quickly. It seemed important to understand the losses well enough to ensure that that observations of cold $\overline{\mathrm{H}}$ were not instead due to $\overline{\mathrm{p}}$ loss induced by $\mathrm{e}^{+}$cooling without $\overline{\mathrm{H}}$ formation. The ambipolar diffusion mechanism [16] was particularly troubling if unbound $\mathrm{e}^{+}$and $\overline{\mathrm{p}}$ could correlate enough to diffuse out of the trap, perhaps even generating simultaneous annihilation of $\overline{\mathrm{p}}$ and $\mathrm{e}^{+}$. These subtle features suggest that great care must be taken when attributing signals observed during the $\mathrm{e}^{+}$cooling of $\overline{\mathrm{p}}$ that go away when there are no $\mathrm{e}^{+}$in the nested Penning trap. Such signals can be generated for reasons other than $\overline{\mathrm{H}}$ production.

After briefly summarizing the methods used to observe cold $\overline{\mathrm{H}}$, we will discuss the important $\overline{\mathrm{p}}$ losses we observed from the nested Penning trap - losses that take place even with no $\mathrm{e}^{+}$present - since some care must be taken to make sure these losses are not confused with $\overline{\mathrm{H}}$ production.

\section{Simultaneous Annihilation Detection - Used by ATHENA, Abandoned by ATRAP}

Cold $\overline{\mathrm{H}}$ formed in the nested Penning trap will leave the trap and hit its electrodes, whereupon both the $\overline{\mathrm{p}}$ and $\mathrm{e}^{+}$will annihilate. The charged pions from $\overline{\mathrm{p}}$ annihilation can be detected with high efficiency with scintillators, scintillating fibers, or silicon strips. The $0.5 \mathrm{MeV}$ gammas from $\mathrm{e}^{+}$annihilation can be detected using CsI or BGO detectors. One might then expect that a simultaneous annihilation of $\mathrm{a} \mathrm{e}^{+}$and a $\overline{\mathrm{p}}$ annihilation would unambiguously demonstrate that an $\overline{\mathrm{H}}$ atom was formed. Un- 
fortunately, we shall see that false $\overline{\mathrm{H}}$ events are unavoidable, and detector resolution limits how accurately the relative position and time of $\overline{\mathrm{p}}$ and $\mathrm{e}^{+}$can be determined.

Clean, event-by-event identification of $\overline{\mathrm{H}}$ is not possible because some $\overline{\mathrm{p}}$ annihilation branches generate secondary $\mathrm{e}^{+}$. This detection method thus unavoidably has a background of false $\overline{\mathrm{H}}$ events which cannot be distinguished from real $\overline{\mathrm{H}}$ events. Some way of estimating the number of true and false signals is thus needed before some of the annihilation signals can be attributed to cold $\overline{\mathrm{H}}$.

Within only a couple weeks of first duplicating our $\mathrm{e}^{+}$cooling of $\overline{\mathrm{p}}$ in a nested Penning trap, ATHENA surprised us by reporting that they had observed cold $\overline{\mathrm{H}}[7]$. (We were carefully studying the subtle losses from the nested Penning trap that we feared could mimic cold $\overline{\mathrm{H}}$ production, intending to publish our own observations at the end of the October $\overline{\mathrm{p}}$ run.) Athena identified $\overline{\mathrm{p}}$ and $\mathrm{e}^{+}$annihilations that took place within $5 \mu$ s and a rather large $\pm 8 \mathrm{~mm}$, as cold $\overline{\mathrm{H}}$. The $131 \pm 22$ events reported were events left after a background slightly larger than the signal was subtracted out.

We look forward to a longer scientific report that gives enough details to allow an evaluation of the short ATHENA announcement. In the next section we discuss concerns about the no-positron control experiment that ATHENA says it relied upon. Our studies of the nested Penning trap suggest an alternate interpretation that should be ruled out. Also, given that $\overline{\mathrm{p}}$ and $\mathrm{e}^{+}$annihilating as far as $\pm 8 \mathrm{~mm}$ and $5 \mu$ s apart would be counted as $\overline{\mathrm{H}}$, arguments and demonstrations that exclude $\overline{\mathrm{p}}$ and $\mathrm{e}^{+}$leaving the trap together in $\overline{\mathrm{H}}$ continuum states will presumably be forthcoming.

At ATRAP we tried similar coincident annihilation detection, though with less axial and energy resolution [17]. The $\overline{\mathrm{p}}$ annihilations were detected in scintillators and scintillating fibers, and $\mathrm{e}^{+}$annihilations were detected in 12 BGO crystals that surrounded the nested Penning trap. Signals remained after subtracting what was seen with no $\mathrm{e}^{+}$in the nested Penning trap. As we shall see, however, our careful studies of $\mathrm{e}^{+}$cooling in the nested Penning trap made us uncomfortable relying upon a no-positron control. Instead we developed a field-ionization method that avoids this difficulty, produces no background of false events, and allows us to learn something about which states of $\overline{\mathrm{H}}$ are produced, as discussed in later sections.

\section{$4 \quad \bar{p}$ Losses from a Nested Penning Trap Invali- date Some No-positron Controls}

$\overline{\mathrm{H}}$ is formed when $\mathrm{e}^{+}$cooling reduces the energy of $\overline{\mathrm{p}}$ to the point where the $\overline{\mathrm{p}}$ move slowly through the central region of the trap where the $\mathrm{e}^{+}$are located. A resulting $\overline{\mathrm{p}}$ loss from the nested Penning trap is very desirable when this loss is due to $\overline{\mathrm{H}}$ production. Neutral $\overline{\mathrm{H}}$ will, of course, be free to drift out of the nested Penning trap unless it is ionized by the fields in this trap on the way out.

Unfortunately, we have observed that $\bar{p}$ of approximate this energy are lost from the nested Penning trap, even when no $\mathrm{e}^{+}$are present. Such losses do not occur when the $\bar{p}$ energy is high enough to keep them from moving slowly over the inverted $\mathrm{e}^{+}$well in the center of the nested Penning trap. As the $\overline{\mathrm{p}}$ energy reduces to where they move slowly over the inverted $\mathrm{e}^{+}$well, however, substantial $\overline{\mathrm{p}}$ losses take place. Our detectors indicate these $\overline{\mathrm{p}}$ escape the trap radially, in directions perpendicular to the magnetic field.

To see these losses requires two conditions. First, there must be enough $\bar{p}$ present so that the "evaporative cooling" mentioned takes place. In collisions, some of the $\overline{\mathrm{p}}$ must 
lose energy and approach the lossy energy regime. Second, since the evaporative cooling mechanism is very slow (much slower than $\mathrm{e}^{+}$cooling) it is necessary to wait a long enough time.

There is even the possibility that such losses might be increased when $\mathrm{e}^{+}$are in the nested Penning trap, insofar as the electrostatic effect of the $\mathrm{e}^{+}$is to flatten the potential that $\overline{\mathrm{p}}$ see as they travel through the central region of the trap. For many years we have observed that low energy particles can escape a trap with a flat potential.

Of course, the major effect of introducing $\mathrm{e}^{+}$into the nested Penning trap is that $\overline{\mathrm{p}}$ are cooled efficiently down to the energies at which $\overline{\mathrm{H}}$ is formed and at which $\overline{\mathrm{p}}$ are lost without $\overline{\mathrm{H}}$ formation. Some way is needed to distinguish the two processes $\overline{\mathrm{p}}$ loss by itself does not indicate the production of $\overline{\mathrm{H}}$.

Notice that if one sees $\overline{\mathrm{p}}$ loss with $\mathrm{e}^{+}$in the nested well, and this $\overline{\mathrm{p}}$ loss goes away without $\mathrm{e}^{+}$, this is not a safe indication of $\overline{\mathrm{H}}$ production. Without $\mathrm{e}^{+}$to efficiently take the $\bar{p}$ to the energies at which the losses occur, it is difficult to even see these losses and to thus know that they exist since this takes a long time and a sufficient number of $\bar{p}$.

One must be particularly careful here when coincident annihilations of $\overline{\mathrm{p}}$ and $\mathrm{e}^{+}$are being identified as potential $\overline{\mathrm{H}}$ signals. As has been discussed, a $\overline{\mathrm{p}}$ annihilation alone can produce a coincident $\mathrm{e}^{+}$annihilation. This means that if the number of coincident annihilation signals increases when $\mathrm{e}^{+}$are put in the nested Penning trap, the simplest interpretation is that $\mathrm{e}^{+}$cooling has reduced the energy of $\bar{p}$ to the point where $\bar{p}$ losses can take place. Some of this loss may be the desired formation of cold $\overline{\mathrm{H}}$, but this is not guaranteed.

The inadequacy of such a no-positrons control experiment kept us at ATRAP from claiming the observation of cold $\overline{\mathrm{H}}$ using this technique, despite our observation that $\mathrm{e}^{+}$in the nested Penning trap increased the number of coincident annihilations of $\overline{\mathrm{p}}$ and $\mathrm{e}^{+}$[17]. Instead, we developed a field ionization technique which avoids this problem, and also allows us to learn about the $\overline{\mathrm{H}}$ states produced, as discussed in the next section.

Unfortunately, the ATHENA announcement relies explicitly upon a no-positron control. A central argument says that the absence of an observed signal in a nopositron control "rules out" the possibility that any "source of antihydrogen-like signal" contaminates their signal. In light of our observations above, however, an alternate no $\overline{\mathrm{H}}$ scenario, not ruled out by the no-positron control, could also explain what is observed. In this scenario, without $\mathrm{e}^{+}$in the nested Penning trap, the $\overline{\mathrm{p}}$ never cool down to the energies at which $\overline{\mathrm{p}}$ loss takes place. Thus no signal is generated in the no-positron control since there is no $\overline{\mathrm{p}}$ loss and hence no false $\overline{\mathrm{H}}$ signals. With $\mathrm{e}^{+}$in the trap in this scenario, the $\overline{\mathrm{p}}$ do cool down to energies at which they can be lost from the nested trap. The observed signal would then be from false $\overline{\mathrm{H}}$ events generated by $\overline{\mathrm{p}}$ loss.

Given that the short ATHENA announcement came only several weeks after they first duplicated our positron cooling in a nested Penning trap, it is likely that they were unaware of the subtle $\overline{\mathrm{p}}$ losses from the nested Penning trap at $\overline{\mathrm{p}}$ energies nearly the same as are needed for $\overline{\mathrm{H}}$ production. ATHENA also does not see the evaporative cooling of $\bar{p}$ that ATRAP used to demonstrate such losses with no $\mathrm{e}^{+}$present, perhaps because they have less $\overline{\mathrm{p}}$ at higher temperature within longer trap electrodes.

Before the observed signals can be reliably attributed to cold $\overline{\mathrm{H}}$, in light of observed $\overline{\mathrm{p}}$ losses from a nested Penning trap, more than the no-positron control argument given 
is needed to rule out the alternate scenario. One help would be an improved control if signal peak is observed when the same number of $\overline{\mathrm{p}}$ used for $\overline{\mathrm{H}}$ production are allowed to radially escape from the trap. Other alternative justifications may be possible and may have emerged during subsequent measurements.

\section{ATRAP's Field Ionization Method}

Fears about the validity of no-positron controls, and the substantial background that cannot be avoided with coincident annihilation detection, prompted ATRAP to develop a field ionization method that avoids these difficulties. ATRAP was able to make a background-free observation of cold $\overline{\mathrm{H}}$ by field ionizing cold $\overline{\mathrm{H}}$ and storing the released $\overline{\mathrm{p}}$ until the $\mathrm{e}^{+}$cooling and its associated background were over [8]. A second ATRAP report demonstrated a method of driving the production of cold $\overline{\mathrm{H}}$ to further increase the production efficiency [9]. Much larger numbers of $\overline{\mathrm{H}}$ were observed per unit time and per high energy $\overline{\mathrm{p}}$ than has been seen to date - more $\overline{\mathrm{H}}$ being observed in an hour than the sum all reported earlier. The field ionization method also made it possible to measure the first distribution of $\overline{\mathrm{H}}$ states, giving the first glimpse inside the $\overline{\mathrm{H}}$ atom. Knowing the internal state of the $\overline{\mathrm{H}}$ is essential if methods to deexcite it to states that can be used for spectroscopy and trapping are to be developed.

For the demonstration of background-free observation of cold $\overline{\mathrm{H}}[8]$, ATRAP typically used $150000 \overline{\mathrm{p}}$ suspended within electrode T2 (Fig. 3a) and up to 1.7 million cold $\mathrm{e}^{+}$in electrode $\mathrm{T} 5$. To start $\mathrm{e}^{+}$cooling and $\overline{\mathrm{H}}$ formation, the $\overline{\mathrm{p}}$ are launched into the nested Penning trap by pulsing from the solid to the dashed potential (Fig. 3b) for $1.5 \mu \mathrm{s}$. The $\overline{\mathrm{p}}$ oscillate back and forth within a nearly symmetrical nested Penning trap, restored before the $\bar{p}$ return to their launch point. They lose energy via collisions as they pass through the $\mathrm{e}^{+}$, which cool via synchrotron radiation to the $4.2 \mathrm{~K}$ of their surroundings.
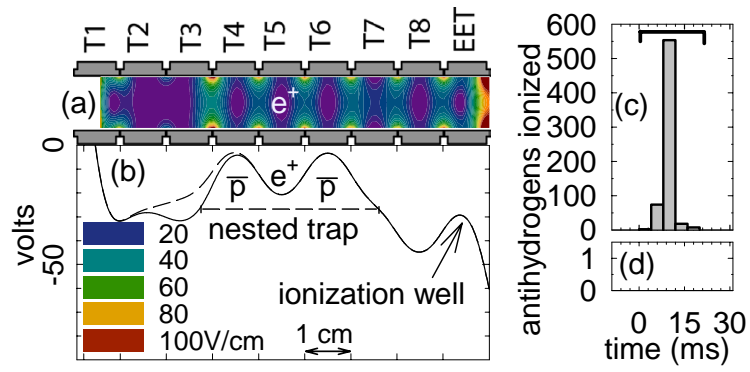

Figure 3: (a) Electrodes for the nested Penning trap have an inner diameter of $1.2 \mathrm{~cm}$. Inside is a representation of the magnitude of the electric field that strips $\overline{\mathrm{H}}$ atoms. (b) The potential on axis for positron-cooling of antiprotons (solid) during which $\overline{\mathrm{H}}$ formation takes place, with the (dashed) modification used to launch $\overline{\mathrm{p}}$ into the well. (c) Antiprotons from $\overline{\mathrm{H}}$ ionization are released from the ionization well during a $20 \mathrm{~ms}$ time window. (d) No background $\overline{\mathrm{p}}$ are counted when no $\mathrm{e}^{+}$are in the nested Penning trap. (From Ref. [8].)

Any $\overline{\mathrm{H}}$ atom formed is free to move in the initial direction of its $\overline{\mathrm{p}}$, unconfined by 
the nested Penning trap. $\overline{\mathrm{H}}$ atoms passing through the field-ionization well in a state that can be ionized by the electric field, will leave their $\overline{\mathrm{p}}$ trapped in this well.

The ionization (or detection) well (within electrode EET in Fig. 3a) is carefully constructed so that its electric field ensures that no $\bar{p}$ from the nested Penning trap can get into it (eg. a $\overline{\mathrm{p}}$ liberated from the nested well by ambipolar diffusion) except if it travels about $4 \mathrm{~cm}$ bound within an $\overline{\mathrm{H}}$ atom. Any $\overline{\mathrm{p}}$ heated out of the nested Penning trap escapes over the lower potential barrier in the other direction. Even if a $\bar{p}$ did acquire enough energy to go over the ionization well in one pass it would not be trapped because there is no mechanism to lower its energy while over this well. In addition, $\mathrm{e}^{+}$cooling lowers the energy of the $\overline{\mathrm{p}}$ in the nested well, taking them further from the energy required to even pass over the ionization well.

Only signals from $\overline{\mathrm{H}}$ are detected with this field-ionization method - there is no background at all! Fig. 3c represents 657 ionized $\overline{\mathrm{H}}$ captured in the ionization well during the course of this experiment - more than all the $\overline{\mathrm{H}}$ atoms reported so far. In many trials without $\mathrm{e}^{+}$we have never seen a single $\overline{\mathrm{p}}$ in the ionization well (Fig. $3 \mathrm{~d}$ ). Antiprotons from $\overline{\mathrm{H}}$ ionization are stored in the ionization well until after positron cooling is completed in the nested well, and all other $\overline{\mathrm{p}}$ and $\mathrm{e}^{+}$are released in the direction away from the ionization well. We then eject the trapped $\bar{p}$ by ramping down the potential of the ionization well in $20 \mathrm{~ms}$. The ejected $\overline{\mathrm{p}}$ annihilate upon striking electrodes, generating pions and other charged particles that produce light pulses in the scintillators. The ramp is fast enough so that the $1.2 / \mathrm{s}$ cosmic ray background contributes a count in our window only 1 time in 50 in Figs. 3b-c. Our experimentally-calibrated detection efficiency corresponds to 1 in 2.7 of the stored $\overline{\mathrm{p}}$ producing a coincidence signal in surrounding scintillators.

The number of ionized $\overline{\mathrm{H}}$ atoms increases with the number of $\mathrm{e}^{+}$in the nested well (Fig. 4a) as might be expected, though this curve is surprisingly insensitive to the total number of $\mathrm{e}^{+}$for larger $\mathrm{e}^{+}$number. We are exploring some indications that the shape of this measured curve is related to a quadratic dependence of the production rate upon $\mathrm{e}^{+}$density. The ionization well can be moved further away from the center of the nested well, using identical electrodes to the right of EET in Fig. 3a. The decrease in the number of ionized $\overline{\mathrm{H}}$ (Fig. 4b) seems consistent with a quadratic dependence on distance, showing that the $\overline{\mathrm{H}}$ angular distribution is broader than the small solid angle subtended by our ionization well. Isotropic $\overline{\mathrm{H}}$ production and a broad $\overline{\mathrm{H}}$ "beam" along the direction of the magnetic field are both consistent with Fig. 4b. More study is required to see if the trajectories of the highly polarizable Rydberg atoms could be significantly modified by the electric and magnetic fields.

To give some idea of how efficiently $\overline{\mathrm{H}}$ atoms are stripped and detected we use one trial in which $8 \mathrm{AD}$ injection pulses are used to accumulate 148000 cold $\overline{\mathrm{p}}, 430000$ cold $\mathrm{e}^{+}$accumulate simultaneously. After the positron cooling of the antiprotons we determine that $66 \overline{\mathrm{H}}$ have field ionized and left their $\overline{\mathrm{p}}$ in the ionization well. This means that we observe about $8 \overline{\mathrm{H}}$ atoms per AD injection pulse, and about 1 $\overline{\mathrm{H}}$ atom per $2200 \overline{\mathrm{p}}$ in the nested well. (For comparison, smaller values of about $1 / 4$ and 1/12000 pertain for the very recent implementation of positron cooling of $\bar{p}$ in a larger trap using more $\mathrm{e}^{+}[7]$, perhaps because of a higher temperature and a higher background gas pressure.)

If the $\overline{\mathrm{H}}$ production at ATRAP is isotropic, then the 657 ionized $\overline{\mathrm{H}}$ would represent nearly 170000 cold $\overline{\mathrm{H}}$. This would mean that a remarkable $11 \%$ of the $\overline{\mathrm{p}}$ in the nested Penning trap are forming $\overline{\mathrm{H}}$ atoms - comprising a substantial portion of the large $\overline{\mathrm{p}}$ losses we have been observing during positron-cooling of antiprotons since this 


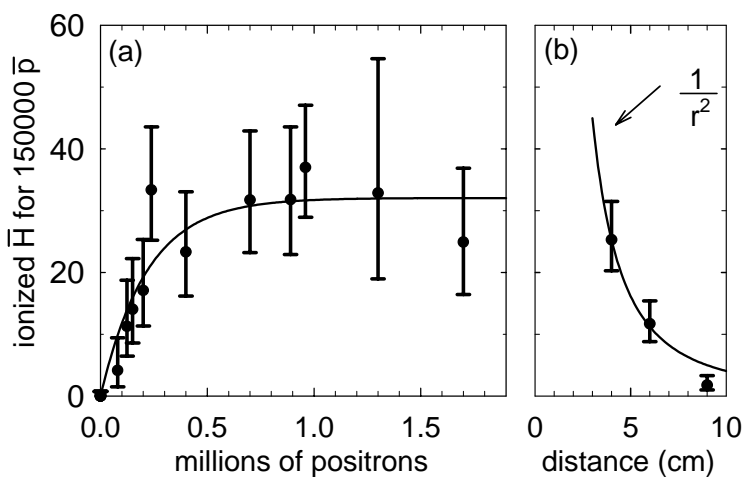

Figure 4: (a) The number of field-ionized $\overline{\mathrm{H}}$ increases with the number of $\mathrm{e}^{+}$in the nested Penning trap of Fig. 3, and then levels off. (b) This number decreases when the ionization well is moved away from the nested Penning trap. (From Ref. [8].)

cooling was first observed. (The ionization well covers only about $1 / 260$ of the total solid angle.)

\section{Driven $\overline{\mathrm{H}}$ Production and the First Measured Distribution of $\overline{\mathrm{H}}$ States}

Knowledge of the $\overline{\mathrm{H}}$ state distributions produced is required to devise methods to prepare states that can be trapped and used for precision spectroscopy. In the second ATRAP observation [9], a distribution of $\overline{\mathrm{H}}$ states is measured for the first time, for a high $\overline{\mathrm{H}}$ production rate realized by driving $\overline{\mathrm{p}}$ into collisions with cold $\mathrm{e}^{+}$.

This time the $\overline{\mathrm{H}}$ states are analyzed as they pass through an electric field that is varied without changing the separated $\overline{\mathrm{H}}$ production and detection. The $\overline{\mathrm{p}}$ are resonantly driven through trapped $\mathrm{e}^{+}$, back and forth from one side of a nested Penning trap to the other, in a new and efficient $\overline{\mathrm{H}}$ production method. $\overline{\mathrm{H}}$ forms during the $\mathrm{e}^{+}$cooling of $\overline{\mathrm{p}}$ over many cycles, until most of the trapped $\overline{\mathrm{p}}$ have formed $\overline{\mathrm{H}}$, or are otherwise lost from the trap. A higher $\overline{\mathrm{H}}$ production rate, per $\overline{\mathrm{p}}$ coming to our apparatus, compensates for the reduced detection solid angle caused by the clean spatial separation of production and detection. The high rate and observed Rydberg states is what would be expected for a three body recombination mechanism [13, 18, 19].

For this observation, the nested Penning trap $[13,14,15,6]$ is again central to $\overline{\mathrm{H}}$ production (Fig. 5a-b). Typically 300000 cold $\mathrm{e}^{+}$are in the central inverted well, with typically $200000 \overline{\mathrm{p}}$ either divided between the two sides of the nested Penning trap (within T6 and T8), or placed in one side well.

The ionization and normalization wells (Fig. 5a-c), to the right and left of the nested Penning trap, are both carefully constructed to prevent $\overline{\mathrm{p}}$ not bound in $\overline{\mathrm{H}}$ from being captured. A $\bar{p}$ heated out of the nested Penning trap will escape over the normalization well, unless there is a mechanism to lower the $\overline{\mathrm{p}}$ energy within this well. To make this harder we keep the potential on the left of this well lower by 3 volts (on axis) 


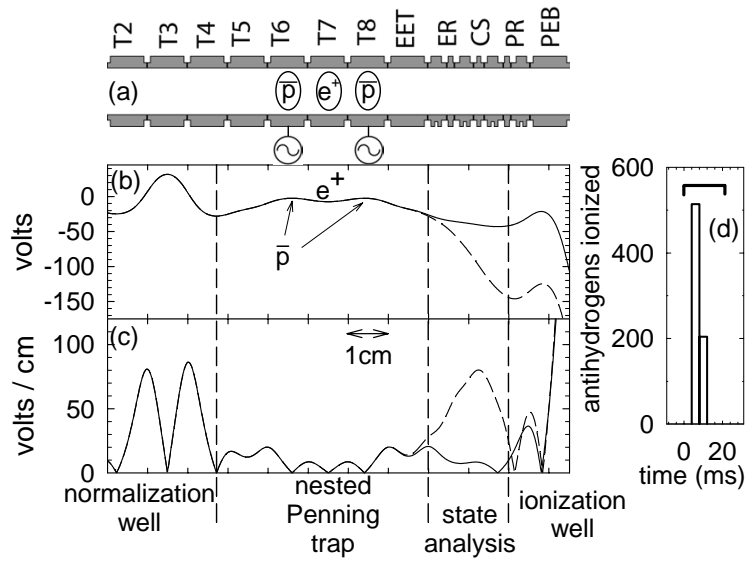

Figure 5: (a) Trap electrodes. (b) Potential on axis. (c) Two values of the electric field magnitude on axis. (d) In a one hour trial $718 \overline{\mathrm{p}}$ from $\overline{\mathrm{H}}$ were captured in the ionization well. (From Ref. [9].)

than that of its right side. Getting a $\overline{\mathrm{p}}$ into the ionization well not only requires an energy loss within the well, but also requires that the $\overline{\mathrm{p}}$ climb a substantial potential barrier. Positron cooling keeps the $\overline{\mathrm{p}}$ from being heated and thus makes it less likely that $\overline{\mathrm{p}}$ will be able to pass through the ionization and normalization wells when $\mathrm{e}^{+}$are in the nested well.

Electric fields within the ionization and normalization wells are made large to ionize $\overline{\mathrm{H}}$ passing through and capture their $\overline{\mathrm{p}}$. Fig. 5a shows the electric field on the trap axis; in the critical state-analysis region, it varies by only about $10 \%$ off the axis. Numerical modelling of $\overline{\mathrm{H}}$ trajectories reveals that $\overline{\mathrm{H}}$ stripped by a field between 25 and $150 \mathrm{~V} / \mathrm{cm}$ leave $\overline{\mathrm{p}}$ in the ionization well, while $\overline{\mathrm{H}}$ stripped between 35 and 140 $\mathrm{V} / \mathrm{cm}$ deposit $\overline{\mathrm{p}}$ in the normalization well.

$\overline{\mathrm{H}}$ state analysis, a central feature of this work, is done by varying the potential offset between the nested and ionization wells. This varies the state-analyzing field that $\overline{\mathrm{H}}$ encounter on their way to the ionization well, as illustrated by two examples in Fig. 5c. Any $\overline{\mathrm{H}}$ stripped by this field is unable to deposit its $\overline{\mathrm{p}}$ in the ionization well, causing the measured number of $\overline{\mathrm{p}}$ in this well, $N$, to decrease. (The stripping field in this well is stronger than are the state-analysis fields.) The number $N_{\text {norm }}$ of $\overline{\mathrm{p}}$ from $\overline{\mathrm{H}}$ ionization in the normalization well provides a normalization.

Crucial radiofrequency drive potentials (1 volt peak-to-peak at $825 \mathrm{kHz}$ ) applied alternatively to electrodes $\mathrm{T} 6$ or T8 (Fig. 5a) drive $\overline{\mathrm{p}}$ between the sides of the nested Penning trap. During each cycle, $\mathrm{e}^{+}$cooling allows the $\overline{\mathrm{p}}$ to settle into the opposite, undriven side well of the nested Penning trap, and some form $\overline{\mathrm{H}}$ during this cooling. Some optimization of the drive frequency and amplitude was done, but most of a large parameter space remains to be explored.

We alternately drive $\bar{p}$ in one side then the other of the nested well for $10 \mathrm{~s}$, with 5 seconds between, up to 25 times. Typically we transfer most $\overline{\mathrm{p}}$ from one side to the other, though asymmetries make it common for a constant remnant of a few ten thousands of $\bar{p}$ to remain in one side well during the whole sequence. The drive cycle 
timing was not optimized.

To detect $\overline{\mathrm{p}}$ deposited in the ionization and normalization wells from $\overline{\mathrm{H}}$ ionization, we ramp down these potential wells in $20 \mathrm{~ms}$, after the driving and associated particle loss are over, just as described before. Fig. 5 d represents a background-free observation of $718 \overline{\mathrm{p}}$ captured in an ionization well from $\overline{\mathrm{H}}$ ionization in a single, one-hour trial. With no $\mathrm{e}^{+}$in the nested well no $\overline{\mathrm{p}}$ form $\overline{\mathrm{H}}$ ionization are detected.

The observed $\overline{\mathrm{H}}$ production rate, per $\overline{\mathrm{p}}$ and per detection solid angle, is up to a factor of 12 greater than that observed using one-time positron cooling of antiprotons [8]. The $\overline{\mathrm{H}}$ rate seems very sensitive to the number of $\mathrm{e}^{+}$in the nested well, unlike what was observed for the one-time cooling. This makes some sense insofar as the driving process continually heats the $\overline{\mathrm{p}}$ and hence the $\mathrm{e}^{+}$they collide with. More $\mathrm{e}^{+}$would transfer this heat more rapidly into synchrotron radiation, and increase $\overline{\mathrm{p}}$ and $\mathrm{e}^{+}$overlap. Here too much parameter space remains to be explored. We presume that the $\overline{\mathrm{H}}$ are cold, insofar as the $\overline{\mathrm{H}}$ is likely made after very effective $\mathrm{e}^{+}$cooling of $\overline{\mathrm{p}}$, but this must also be checked.

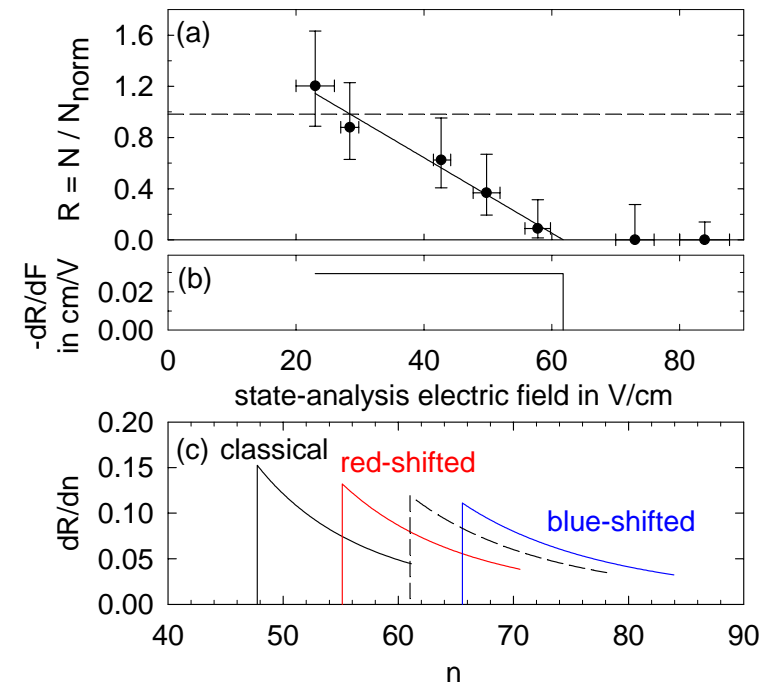

Figure 6: (a) The ratio of ionized $\overline{\mathrm{H}}$ in ionization and normalization wells decreases linearly with state-analysis electric field $F$. (b) Distribution $\mathrm{dR} / \mathrm{dF}$ is flat up to a cutoff. (c) The distribution $d R / d n$ depends upon the relation of $F$ and $n$. (From Ref. [9].)

The first measured distribution of $\overline{\mathrm{H}}$ states is displayed in Fig. 6a. The ratio $(R)$, of the number of $\overline{\mathrm{p}}$ from $\overline{\mathrm{H}}$ stripped in the ionization well $(N)$ to the corresponding number in the normalization well $\left(N_{\text {norm }}\right)$, is plotted as a function of the state-analysis field $(F)$. The number of $\overline{\mathrm{H}}$ that survive this field decreases linearly until consistent with zero. The error bars prevent seeing curvature near this point, so we use simple linear dependence going to zero to explore principal features. Thus $d R / d F$ (Fig. $6 \mathrm{~b}$ ) is constant up to a cutoff. As many $\overline{\mathrm{H}}$ states are ionized by fields between 30 and $35 \mathrm{~V} / \mathrm{cm}$ as between 55 and $60 \mathrm{~V} / \mathrm{cm}$, for example. No observed $\overline{\mathrm{H}}$ states require a 
stripping field greater than $62 \mathrm{~V} / \mathrm{cm}$.

It would be more satisfying to characterize the distribution of $\overline{\mathrm{H}}$ excited states by their principle quantum number $n$, rather than by the electric field that strips them. The first difficulty is that $n$ is not a good quantum number in the strong magnetic field, though we still use $n$ as a rough parameterization of binding energy, using $E=-13.6 \mathrm{eV} / \mathrm{n}^{2}$. Ionization likely takes place in the direction of the magnetic field [20], giving some hope that it may not be strongly modified by the magnetic field, but this must be investigated.

The second difficulty is that the types of states formed determine the electric field that will ionize them, even in the absence of any magnetic field, as is well known from studies of the ionization of Rydberg atoms [21]. Fig. 6c shows $n$ distribution for various models, which we have described in more detail [9]. Fortunately, classical and quantum calculations are underway.

Further enhancements of $\overline{\mathrm{H}}$ production might be possible with optimizations and variations on our method of arranging for many cycles of positron cooling of antiprotons. One might be to simultaneously drive $\overline{\mathrm{p}}$ on both sides of the nested Penning trap. Another would be to lift $\overline{\mathrm{p}}$ from the bottom of the nested well in a potential "bucket" for launching back into the nested Penning trap.

\section{Conclusions and Acknowledgements}

In conclusion, ATRAP's positron cooling of cold antiprotons [6] produces cold antihydrogen. ATHENA [7] and ATRAP [8, 9] both report the production of cold $\overline{\mathrm{H}}$ using this device and technique. The observed $\overline{\mathrm{H}}$ production is encouragingly high, especially when we drive the $\overline{\mathrm{p}}$ into collisions with $\mathrm{e}^{+}$in a nested Penning trap.

ATRAP's field ionization method allows a background-free observation of cold antihydrogen. It also allowed ATRAP to measured the first distribution of $\overline{\mathrm{H}}$ states using a pre-stripping electric field in a separate region between where the $\overline{\mathrm{H}}$ are produced and detected. The observed distribution is constant as a function of the state-analysis field, up to cutoff. The Rydberg states and high production rate are consistent with a three body recombination mechanism [13, 18, 19].

The high production rate and measured state distribution give hope that it may be possible to devise a way to deexcite Rydberg atoms with a range of binding energies and still get enough atoms for trapping and spectroscopy. Some temporary confinement of these highly polarizable states may be possible, but conventional trapping awaits deexcitation to the ground state, whereupon a goal is to superimpose a magnetic trap for $\overline{\mathrm{H}}$ with the Penning traps needed for its $\overline{\mathrm{p}}$ and $\mathrm{e}^{+}$ingredients [22]. The ATRAP field ionization method can be extended to more deeply bound states by increasing the size of the electric field. For states not accessible in this way, an excitation laser can be used to excited the deeply bound states to where they can be ionized and stored in the same way.

Thanks to CERN, its PS Division and the AD team for delivering 5.3 MeV antiprotons. ATRAP is supported by the NSF, AFOSR, the ONR of the US, the BMBF, MPG and FZ-J of Germany, and the NSERC, CRC and PREA of Canada. 


\section{References}

[1] G. Gabrielse, in Fundamental Symmetries, edited by P.Bloch, P. Paulopoulos, and R. Klapisch (Plenum, New York, 1987), p. 59.

[2] G. Gabrielse, Adv. At. Mol. Opt. Phys. 45, 1 (2001).

[3] G. Gabrielse, N. S. Bowden, P. Oxley, A. Speck, C. H. Storry, J. N. Tan, M. Wessels, D. Grzonka, W. Oelert, G. Schepers, T. Sefzick, J. Walz, H. Pittner, T. W. Hänsch, and E. A. Hessels, Phys. Lett. B 548, 140 (2002).

[4] G. Baur, et al., Phys. Lett. B 368, 251 (1996).

[5] G. Blanford, D. C. Christian, K. Gollwitzer, M. Mandelkern, C. T. Munger, J. Schulz, and G. Zioulas, Phys. Rev. Lett. 80, 3037 (1998).

[6] G. Gabrielse, J. Estrada, J. N. Tan, P. Yesley, N. S. Bowden, P. Oxley, T. Roach, C. H. Storry, M. Wessels, J. Tan, D. Grzonka, W. Oelert, G. Schepers, T. Sefsick, W. Breunlich, M. Carngelli, H. Fuhrmann, R. King, R. Ursin, H. Zmeskal, H. Kalinowsky, C. Wesdorp, J. Walz, K. S. E. Eikema, and T. W. Hänsch, Phys. Lett. B 507, 1 (2001).

[7] M. Amoretti, et al., Nature 419, 456 (2002).

[8] G. Gabrielse, N. S. Bowden, P. Oxley, A. Speck, C. H. Storry, J. N. Tan, M. Wessels, D. Grzonka, W. Oelert, G. Schepers, T. Sefzick, J. Walz, H. Pittner, T. W. Hänsch,and E. A. Hessels, Phys. Rev. Lett. 89, 213401 (2002).

[9] G. Gabrielse, N. S. Bowden, P. Oxley, A. Speck, C. H. Storry, J. N. Tan, M. Wessels, D. Grzonka, W. Oelert, G. Schepers, T. Sefzick, J. Walz, H. Pittner, T. W. Hänsch,and E. A. Hessels, Phys. Rev. Lett. 89, 233401 (2002).

[10] R. Bluhm, V. A. Kostelecký, and N. Russell, Phys. Rev. D 57, 3932 (1998).

[11] M. Niering, R. Holzwarth, J. Reichert, P. Pokasov, T. Udem, M. Weitz, T. W. Hänsch, P. Lemonde, G. Santarelli, M. Abgrall, P. Laurent, C. Salomon, et al., Phys. Rev. Lett. 84, 5496 (2000).

[12] G. Gabrielse, Hyper. Int. 44, 349 (1988).

[13] G. Gabrielse, S. L. Rolston, L. Haarsma, and W. Kells, Phys. Lett. A129, 38 (1988).

[14] D. S. Hall and G. Gabrielse, Phys. Rev. Lett. 77, 1962 (1996).

[15] G. Gabrielse, D. S. Hall, T. Roach, P. Yesley, A. Khabbaz, J. Estrada, C. Heimann, and H. Kalinowsky, Phys. Lett. B 455, 311 (1999).

[16] R. J. Goldston and P. H. Rutherford, Introduction to Plasma Physics (Inst. of Phys., London, 1995).

[17] To be published.

[18] M. Glinsky and T. O'Neil, Phys. Fluids B3, 1279 (1991).

[19] P. O. Fedichev, Phys. Lett. A 226, 289 (1997).

[20] W. Ihra, F. Mota-Furtado, and P. F. O’Mahony, Phys. Rev. A 58, 3884 (1998).

[21] T. F. Gallagher, Rydberg Atoms (Cambridge Univ. Press, New York, 1994).

[22] T. M. Squires, P. Yesley, and G. Gabrielse, Phys. Rev. Lett. 86, 5266 (2001). 